\title{
Les catholiques et l'argent. Une approche de la paroisse par ses finances
}

Nicolas de Bremond d'Ars

\section{(2) OpenEdition \\ 1 Journals}

Édition électronique

URL : http://journals.openedition.org/assr/3386

DOI : 10.4000/assr.3386

ISSN : $1777-5825$

Éditeur

Éditions de l'EHESS

\section{Édition imprimée}

Date de publication : 1 mars 2006

Pagination : 67-92

ISBN : 2-7132-2046-7

ISSN : 0335-5985

Référence électronique

Nicolas de Bremond d'Ars, «Les catholiques et l'argent. Une approche de la paroisse par ses finances », Archives de sciences sociales des religions [En ligne], 133 | janvier - mars 2006, mis en ligne le 03 mai 2009, consulté le 19 avril 2019. URL : http://journals.openedition.org/assr/3386 ; DOI : 10.4000 /assr.3386 


\section{Nicolas de Bremond d'Ars}

\section{Les catholiques et l'argent. Une approche de la paroisse par ses finances}

Le catholicisme en France se déploie en de nombreuses configurations, institutionnelles ou non, héritées d'une histoire très ancienne ou récente. Les paroisses en constituent le paysage le plus visible, parce que le monopole de distribution des sacrements qu'elles administrent les rend incontournables pour le peuple catholique. Issues des découpages successifs du territoire français, en vue d'assurer le contrôle des autorités publiques aussi bien que celui des évêques, elles disposent d'une autonomie juridique et véhiculent l'image la plus archétypale de la présence du catholicisme en France : à chaque village son clocher ${ }^{1}$. Du fait de cette position stratégique, la vitalité du catholicisme est largement tributaire de celle des paroisses. La crise démographique qui affecte actuellement le personnel clérical secoue depuis plus de vingt ans l'arbre pluriséculaire des paroisses, avec une ardeur accrue en ce début de siècle. En même temps, la transformation interne des objectifs d'animation ${ }^{2}$ et des modalités de contrôle religieux ${ }^{3}$ conduit à un investissement massif des laïcs dans le fonctionnement de ces paroisses. Les modalités de la vitalité du catholicisme sont donc en train de se transformer.

L'objectif de cet article est de présenter l'intérêt, pour l'appréhension de la vie interne des paroisses catholiques, d'une approche par l'analyse financière

1. Le Droit Canon de 1983 définit la paroisse par la population, et non par le ressort géographique : "Sur le plan juridique, les paroisses sont des parties du diocèse, que l'évêque est obligé de constituer à partir de critères permettant de déterminer des communautés de fidèles nécessitant une prise en charge pastorale. Les éléments constitutifs des communautés hiérarchiques se retrouvent tous ici, en premier lieu l'élément communautaire, dont la détermination n'est pas liée, bien qu'il reste traditionnellement prépondérant, à l'utilisation du critère de territorialité. On peut en effet constituer des paroisses personnelles selon le rite, la langue, la nationalité, et pour d'autres motifs. Dans tous les cas, comme pour la délimitation des Églises particulières, notamment du diocèse, l'appartenance est de type objectif. » Précis Dalloz, Droit canonique, $2^{\mathrm{e}}$ édition, 1999, p. 169.

2. Parmi ceux-ci, signalons la profonde transformation des enseignements catéchétiques depuis plus de trente ans, qui a pour effet de changer le corpus des croyances des catholiques français contemporains.

3. En particulier l'ouverture aux laïcs de la présidence d'assemblées de prière et des obsèques, et l'apparition des diacres pour la célébration des baptêmes et mariages.

ARCHIVES DE SCIENCES SOCIALES DES RELIGIONS 133 (janvier-mars 2006), p. 67-92 
des flux monétaires qui les traversent ${ }^{4}$. En effet, de nombreuses activités paroissiales font l'objet d'une transaction monétaire : catéchèse des enfants, kermesses, cérémonies, solidarité, etc., sans compter le soutien financier des paroissiens à l'institution (quêtes, Denier de l'Église [DDE]). À l'autre extrémité, les paroisses dépensent cet argent reçu de façon à perpétuer leur service, tout en orientant les choix religieux pratiques. Le diocèse, qui est la division administrative de base, avec l'évêque à sa tête, s'intercale dans la gestion des flux selon différentes configurations. L'écheveau n'est pas si embrouillé qu'il ne puisse faire l'objet d'une analyse sociologique au cas par cas. Mais nous cherchons ici à les comprendre comme une réalité complexe (des flux en interdépendance) qui rend compte des logiques religieuses présentes dans le catholicisme quotidien. En ce sens, il ne s'agit ni du catholicisme militant, ni de l'étude des croyances ayant cours dans une opinion catholique, ni même d'une recherche sur le contrôle normatif d'une Église romaine.

Dans un premier temps nous présenterons la méthode suivie, de façon ramassée. Elle a été élaborée à partir de six paroisses à Paris et en province, et rien des recherches ultérieures (hormis les inévitables changements de normes comptables et de pratiques institutionnelles) ne permet à ce jour d'en infirmer la pertinence ${ }^{5}$. La deuxième partie sera consacrée à une présentation pratique des recettes des paroisses, avec une insistance sur les dons des pratiquants occasionnels. En troisième lieu nous analyserons succinctement deux budgets paroissiaux l'un dans le Sud-Ouest, l'autre à Paris. Nous verrons que les comportements des catholiques s'avèrent particulièrement convergents malgré les différences culturelles et fonctionnelles. Le quatrième point mettra en perspective le don des catholiques pratiquants et le don en France.

\section{Méthode et outils}

Le budget paroissial, présenté selon les normes comptables habituelles des associations, réunit l'ensemble des flux, recettes et dépenses, qui transitent par l'église paroissiale et ses alentours institutionnels. Le décryptage de ces flux s'appuie sur les règles de la comptabilité analytique, à laquelle il faut adjoindre des clés de lecture sociologiques et anthropologiques. Nous voudrions décrire ici les procédures et méthodes qui nous ont été indispensables ${ }^{6}$. Deux remarques préalables sont cependant nécessaires.

4. Une approche par les flux se distingue d'une approche par les stocks en ce qu'elle considère d'abord la relation établie comme signifiante, et secondairement les montants.

5. Pour des raisons d'homogénéité, la comparaison doit s'établir sur des périodes identiques. Notons en particulier les changements de comptabilité et de fiscalité qui interviennent dans le cours des années 1990.

6. L'ensemble des données de l'enquête (en particulier la description des paroisses) est présenté dans Nicolas DE BREMOND D'ARS, Dieu aime-t-il l'argent? Don, piété et utopie sociale chez les catholiques en France, Paris, L'Harmattan 2006. 
Dès que l'on veut aborder la dimension financière, on est contraint de s'affronter à la comptabilité, qui est un système organisé. Elle obéit à des règles qui donnent leur lisibilité aux flux monétaires et, de ce fait, en prédéterminent la circulation. Par la comptabilité, des réalités hétérogènes sont intégrées dans une même structure. Les techniques d'analyse financière donnent ensuite des clés de lecture, non pas pour comprendre les données indépendamment les unes des autres, mais au contraire en interaction. La combinaison et le retraitement des rubriques et des chiffres offrent enfin un éclairage inédit sur les logiques implicites de faits observés.

Nous livrons ici le résultat d'un tel travail. Il porte sur quelques paroisses, il est extensible à la plupart des paroisses françaises. En effet, si le terrain est circonscrit, l'homogénéité des règles comptables et l'universalité des pratiques décrites (recettes et dépenses) lui donnent un caractère d'exemplarité.

Le deuxième enjeu de cette approche est de respecter le contexte culturel qui rend efficient une régulation économique des pratiques religieuses. Par là nous entendons que l'usage des dons d'argent dans la société française de la fin du $\mathrm{Xx}^{\mathrm{e}}$ siècle ne peut pas être du même ordre que dans la France du $\mathrm{XvI}^{\mathrm{e}}$ siècle ${ }^{7}$, ni dans des sociétés nord-américaines, par exemple, où la transaction monétaire ne véhicule pas les mêmes a priori idéologiques ${ }^{8}$. Ce n'est pas le lieu de cet article que de le présenter; à tout le moins le chercheur doit-il prendre en compte cette dimension dans l'interprétation qu'il tente d'une approche par les flux monétaires. L'Église catholique en France est financée très majoritairement par les dons des fidèles (à l'exception du patrimoine immobilier pour une large part - mais il s'agit d'une question complexe). Or, comme le montrent les études menées par la Fondation de France, les dispositifs fiscaux jouent un rôle non négligeable sur le montant des dons. Par ailleurs, donner de l'argent est un usage parmi d'autres dans la société monétaire - le commerce des «biens de salut » étant une autre possibilité.

Les comptes d'exploitation sont la meilleure et première source d'information en matière de circulation de l'argent. Leur présentation obéit à des règles comptables et fiscales échappant pour une large part aux nécessités de recherche du sociologue. D'une part il faut remarquer, en effet, que bien des recettes affluent sous forme d'espèces, ce qui rend difficile toute traçabilité une fois enregistrées (comme pour toute activité commerciale). Pour deux paroisses, nous avons reconstitué les totaux à partir du journal comptable qui rassemble les données quotidiennes, et reconstruit des séries de recettes: les quêtes dominicales comptées le mardi additionnaient les quêtes faites lors des messes, celles à l'occasion des cérémonies autres, et troncs des cierges. Les entretiens avec les responsables permettent de déjouer ce genre de pratiques, par ailleurs tout à fait légitimes.

7. Natalie Zemon Davis, Essai sur le don dans la France du XVI siècle, Paris, Seuil, 2003.

8. La dimension contextuelle est particulièrement flagrante dans Alain TESTART, Éléments de classification des sociétés, Paris, Errance, 2005. 
D'autres documents sont aussi nécessaires ${ }^{9}$. Ainsi pour l'argent correspondant aux intentions de messes ${ }^{10}$, nous avons rapproché les montants globaux des cahiers de sacristie où elles sont notées journellement, pour avoir la répartition entre intentions pour défunts et les autres. À St Jules ${ }^{11}$, paroisse parisienne, cela représente plus de 1300 inscriptions annuelles en 1990. En outre, comme les paroisses tiennent des fichiers nominatifs (en général disjoints les uns des autres), il est indispensable de procéder à un pointage des noms - si les responsables le permettent - de façon à déterminer si les fidèles donnent à une seule reprise ou bien à plusieurs occasions. Les responsables eux-mêmes nous ont éclairé sur la signification des montants, et sur les identités des donateurs; dans tel cas, il s'agissait de pluri-donateurs, parisiens versant leur DDE à Paris et en province. Les progrès de l'informatique ont également permis aux paroisses de connaître de façon de plus en plus précise la rotation des fidèles. Tel vice-président de conseil économique du Val-de-Marne (diocèse de Créteil) estime le taux de rotation dans sa commune (paroisse) à $20 \%$ par an sur la période 1993-2003.

À ces documents administratifs il convient d'ajouter, si possible, les comptesrendus des séances des différents conseils - conseil économique, conseil pastoral, etc. -, de façon à obtenir les tenants et aboutissants des discussions ayant affecté tant les recettes que les dépenses. On apprend au détour d'un conseil que le tarif "recommandé » pour les cierges passe de $1,50 \mathrm{~F}$ à $2 \mathrm{~F}$, ce qui explique l'augmentation des recettes dans l'exercice suivant. Ou bien encore qu'une délicate affaire de détournement de fonds a eu lieu telle année, portant sur des dizaines ou des centaines de milliers de francs.

Les schémas d'organisation sont différents selon les diocèses : cela implique une gestion différente des ressources, ce qui impose une correction pour rétablir la comparabilité des données financières. On ne saurait comparer les DDE de bien des diocèses de province, collectés par les diocèses directement, et les DDE des diocèses où la gestion en est laissée à la discrétion des paroisses, comme à Paris et la petite couronne. Ainsi, à St Jacques de Grosport (province), les responsables paroissiaux ont créé une "cotisation paroissiale " de façon à faire droit à une revendication de don de proximité de la part des fidèles : le DDE s'en est aussitôt trouvé minoré. Parfois, comme à Montpellier, le bulletin officiel du diocèse publiait les « rendements » du DDE par paroisse d'origine (jusqu'au début des années 1990), dans le but de stimuler les curés! Dans tel diocèse, il existe une sorte d'impôt sur les paroisses, selon des barèmes précis. Ailleurs, il

9. Ce type de travail n'invalide en aucun cas la pertinence de la comptabilité. Il s'agit simplement de la méthode habituelle de l'analyse financière, dont le sociologue n'est pas obligatoirement informé.

10. À titre indicatif, dans un sanctuaire comme Saint-Louis d'Antin à Paris, la paroisse comptabilise environ 4200 euros par mois, soit près de 28000 francs, en 2004.

11. Les noms ont été changés pour respecter une demande de discrétion faite par les différents responsables. 
s'agit d'une taxation recette par recette (comme une TVA), ce qui peut tendre à accroître les procédures de soustraction par dissimulation, puisqu'il s'agit pour l'essentiel d'espèces.

L'étude des documents administratifs, complétée par les entretiens avec les responsables, n'est pas suffisante. Des observations complémentaires aident à clarifier les phénomènes. Nous avons étudié en particulier : les procédures de présentation des frais pour les cérémonies (obsèques, baptêmes et mariages essentiellement) ; les micro-pratiques de paiement des intentions de messe, aux accueils des églises; les modalités des quêtes lors des messes dominicales; les tracts émis pour les campagnes de DDE; le dispositif pratique des cierges de dévotion (associé à des enquêtes et entretiens); et enfin l'environnement socioculturel des paroisses.

\section{Les recettes des paroisses ${ }^{12}$ : commerce ou dons?}

Les budgets paroissiaux sont alimentés de façon permanente par deux types de dons : des flux anonymes plus ou moins importants, d'une part, pour lesquels la régularité dépend de facteurs échappant bien souvent au pouvoir du clergé comme des responsables laïques. Il s'agit des troncs pour les cierges, puisque leur accès est libre, des honoraires de messe, des activités et fêtes, et, dans une moindre mesure, des quêtes. D'autre part, des dons versés à l'occasion des cérémonies (casuel) et le DDE sont pour l'essentiel nominatifs.

\section{Les troncs}

Toute église dispose dans son enceinte d'un ou plusieurs lieux de prière comportant généralement une statue. La Vierge Marie est bien sûr à l'honneur, mais sainte Rita (patronne des causes désespérées) et saint Antoine (pour les objets perdus) conservent une faveur populaire très étendue. Ces espaces comportent la plupart du temps un porte-cierges où brûlent du matin au soir les cierges offerts par les fidèles. Ceux-ci sont disponibles dans des présentoirs, un prix indicatif est affiché et les gens déposent une offrande (mot officiel) dans un tronc mis à proximité. Le don est libre, mais les observations empiriques montrent que peu de gens se soustraient à l'obligation de payer.

Le montant des dons par individu est difficile à apprécier ; nous ne disposons en général que du montant global collecté annuellement (sauf si on dispose du tonnage de cierges écoulés). Cela peut aller de quelques milliers à plusieurs centaines de milliers d'euros, selon la notoriété ou la qualité de l'emplacement de l'église. Le montant est aussi influencé par les événements qui rythment la vie

12. Il s'agit en premier lieu des paroisses de l'enquête. Mais la généralisation est légitime en raison de la diffusion des pratiques. Par exemple, sur 110 paroisses à Paris, toutes usent de ces recettes (les exceptions portent sur un ou deux types, absents ou inusités pour des raisons locales spécifiques). 
sociale (baccalauréat, troubles publics). La modification du prix « indicatif » par les responsables paroissiaux a un impact direct sur les montants collectés. Le scrupule pour payer, selon nos observations, est une donnée intrinsèque à l'acte même.

Le profil des donateurs est difficile à cerner. La zone de chalandise, qui détermine les populations susceptibles d'être concernées, rend l'observation statistique complexe à mettre en œuvre. Il faut également tenir compte des contextes socioculturels de ces populations. Il est certain que les observations ne révèlent pas un profil type du donateur, même si le clergé y voit une dévotion "populaire " à laquelle lui-même se prête de mauvaise grâce ${ }^{13}$.

\section{Les quêtes}

La quête est la pratique financière la plus repérable dans l'imaginaire catholique. Le mot est très naturellement assimilé à l'image des "quêteurs " passant dans les rangs des fidèles, au cours d'un office religieux, pour recueillir les dons. Il signifie également cette sollicitation à l'extérieur des églises, sous le porche lorsqu'il s'agit d'œuvres catholiques, ou dans la rue pour une cause humanitaire. Les mariages à la mairie donnent également lieu à une quête effectuée par un employé municipal, au moins pour le cas de Paris. Nous distinguerons les quatre types suivants, qui sont classés en fonction des lieux, des populations et des modes de fonctionnement : les quêtes du dimanche au cours des messes, au cours des offices autres que la messe (baptêmes, mariages, enterrements), les quêtes aux portes de l'église et celles effectuées dans la rue (voie publique). Pour la commodité de l'exposé, nous considérerons les deux premiers types, les seuls à nous intéresser dans le cadre de l'étude.

La quête qui a lieu à l'intérieur des églises est traditionnellement menée au cours d'une cérémonie : messe dominicale, obsèques, baptême, mariage. L'absence de règles et de processus définis s'imposant aux curés explique une assez grande variété de pratiques. Des fidèles parcourent les rangs, une corbeille à la main, et l'on y dépose pièces ou billets. Certaines paroisses ont supprimé la quête et ont disposé des paniers aux portes de l'église. Pour les cérémonies autres que la messe hebdomadaire, les quêtes sont loin d'être systématiques, car il est d'usage d'indiquer aux personnes qui demandent l'acte cultuel une participation aux frais ${ }^{14}$. De ce fait, les paroisses ne sont pas toujours désireuses d'ajouter à ce versement une quête dans l'assemblée, bien que le montant donné soit à la discrétion du donateur. On trouve plus fréquemment une corbeille à la sortie de la cérémonie, avec, si ce sont des obsèques, une mention "messes pour le défunt ". Il est à noter qu'il arrive aux fidèles eux-mêmes de réclamer parfois une corbeille et de procéder à la quête quand l'officiant a oublié de la disposer.

13. Saint-Jacques de Grosport avait supprimé les troncs et les cierges pour cette raison.

14. Le diocèse de Poitiers a demandé que les baptêmes ne soient pas tarifés (2004). 
Toutes les quêtes n'ont pas la même destination, et la pratique ancienne le manifestait plus clairement qu'aujourd'hui. Au moment de la quête, il n'était pas rare de voir circuler deux paniers successifs; le premier était affecté aux besoins paroissiaux, le second bénéficiait à une œuvre ou cause quelconque, et notamment à celles recommandées par l'évêque. Lorsque, comme c'est le cas communément aujourd'hui, un seul panier de quête circule, la coutume veut que l'annonce de la destination des fonds recueillis la précède : " aujourd'hui la quête est pour..." et que s'applique alors un principe de partage, variable selon les paroisses. En règle générale, la paroisse conserve un montant qu'elle juge conforme à une quête ordinaire et affecte tout le supplément à l'œuvre bénéficiaire, mais on connaît des cas où le partage se fait par moitié.

Le don moyen par pratiquant apporte un complément de données indispensable à l'explication du comportement des fidèles. Sur la base des estimations de pratique dominicale, avec toutes les corrections qu'il nous a été possible de faire, pour l'année 1994, le don moyen calculé dans les six paroisses de l'enquête allait de $5,35 \mathrm{~F}$ à $5,95 \mathrm{~F}^{15}$.

La quête n'est donc pas un rite économique, car l'augmentation considérable du niveau de vie en cinquante ans a relégué au rang de pratique ancestrale le passage du panier. Une observation précise du geste pratiqué par les fidèles, que ce soit au cours d'un office ou à la sortie de l'église, révèle la prégnance de la main mise à la poche ou dans le sac à main. Une corrélation étroite existe entre la fréquence des pièces disponibles dans la poche (ou le porte-monnaie) et le contenu des paniers de quête. La fréquence de la monnaie divisionnaire (les pièces) disponible sur le marché conditionne fortement le don moyen.

La quête manuelle relève donc d'un aspect fondamental du groupe social catholique : la transmission du geste de l'aumône. Le comportement des familles qui viennent à la messe dominicale est, sur ce point, très instructif : les enfants (surtout les moins de douze ans) reçoivent de leurs parents des pièces de monnaie pour imiter le geste de tous. Si un enfant n'a pas vu passer la corbeille, il n'est pas rare de le voir se lever et parcourir quelques mètres pour rejoindre un quêteur et donner sa pièce. L'enfant apprend à donner, et saura, à son tour, poursuivre cet acte tellement valorisé par le groupe social éducateur. On comprend aussi pourquoi des personnes aux revenus élevés ne glissent que des pièces rouges (centimes) à la quête : l'inertie de l'apprentissage pendant l'enfance se manifeste à plein, au grand dam des curés parfois !

15. Ces chiffres comprennent les minimes retraitements comptables en vue de rendre possible les comparaisons. Autre indication: La Baule (Loire-Atlantique), août 1997, quête moyenne pour 3500 personnes : $20000 \mathrm{~F}$, soit 5,71 F/pers. La variable « région culturelle » n'est donc pas pertinente. 


\section{Les intentions de messe}

Les églises catholiques disposent d'une troisième ressource, autrefois indispensable, qui ne représente plus aujourd'hui qu'un élément marginal du budget paroissial, les honoraires de messe ${ }^{16}$. Comme son nom l'indique, l'honoraire est une somme d'argent versée à l'occasion d'une messe par une personne qui souhaite que l'intention qui la préoccupe soit prononcée au cours de l'office.

Guillaume Cuchet a présenté de façon magistrale la genèse et le " crépuscule » des intentions de messe en lien avec des préoccupations de salut, aux $\mathrm{XIX}^{\mathrm{e}}$ et $\mathrm{XX}^{\mathrm{e}}$ siècles ${ }^{17}$. Le recyclage du purgatoire, entre le Ciel et l'Enfer, dans les préoccupations liées au devenir des défunts, a permis à l'Église catholique d'associer plus étroitement intentions de messe et inquiétude populaire. La Grande Guerre a parachevé le déclin entamé au début du $\mathrm{XX}^{\mathrm{e}}$ siècle. Le dispositif que nous avons analysé est donc à la fois un reliquat de ce système et la perdurance d'une pratique de piété liée à la messe. Trois générations après le début du déclin des intentions de messe en faveur des âmes du purgatoire, les intentions pour les défunts et pour diverses circonstances demeurent.

L'argent est versé par les demandeurs le plus souvent en espèces. Dans les deux tiers des cas environ il concerne des défunts, puis viennent les intentions pour « obtenir une grâce ». La présence du demandeur n'est pas indispensable, bien qu'elle soit conseillée. La plupart du temps, les intentions sont demandées aux services d'accueil des églises ou aux secrétariats, et l'argent est versé directement dans la caisse paroissiale.

Il faut noter en outre que dans les sociétés humaines à forte surveillance, les honoraires permettaient à chacun de compter ses alliés. Ainsi, dans les paroisses du Léon (Finistère) comme dans bien d'autres régions de France, il était d'usage d'annoncer les intentions demandées au cours de la cérémonie d'obsèques. En fin de liturgie, le curé énonçait les demandeurs : «famille N..., quinze messes ». Certains fins observateurs locaux nous ont rapporté qu'à ce moment les personnes âgées des premiers rangs égrenaient les intentions sur leurs chapelets, transformés provisoirement en bouliers... Le défunt était important ou sa famille puissante en fonction du nombre d'intentions souscrites à ce moment.

16. Pour ce qui concerne les églises paroissiales. Les grands sanctuaires comme Lourdes ou le Sacré-Cœur de Montmartre disposent encore d'un montant appréciable d'honoraires, qu'ils redistribuent ensuite en d'autres lieux.

17. Guillaume Cuchet, Le crépuscule du purgatoire, Paris, Armand Colin, 2005. Le chapitre 8 fournit de précieuses indications sur le cadre institutionnel et financier. Nous ne reprendrons donc pas ici ces données fort bien exposées. 


\section{Activités et fêtes}

Une paroisse organise traditionnellement des rassemblements festifs périodiques, parfois lors de la fête patronale ou bien le plus souvent à une date choisie en accord avec les paroisses voisines. À Paris, le vocable le plus fréquemment utilisé est celui de " journées d'amitié ", indiquant par là l'intention officielle de cette mobilisation. En réalité, il s'agit dans la plupart des cas de ce qu'on appelait autrefois " vente de charité ": comptoirs divers (brocante, livres, vêtements, etc.) repas animés ou non, soirées de jeux de société ou de spectacle, etc. ${ }^{18}$. Des deux objectifs poursuivis : l'animation festive de la vie paroissiale et les rentrées de fonds, le second est à l'évidence prioritaire. Ces rentrées financières constituent bien souvent un appoint tout à fait conséquent aux quêtes et autres cotisations, et peu nombreux seraient les curés qui accepteraient de tirer un trait sur ce commerce fructueux.

D'une kermesse à l'autre les mêmes profils de personnes âgées sont installés pour tenir les mêmes comptoirs. La mobilisation des clients s'effectue alors par le bouche à oreille, les responsables étant les meilleurs recruteurs lorsqu'ils donnent rendez-vous à leurs amis et connaissances. Hormis les brocanteurs professionnels qui se pressent dès la première heure d'ouverture, la quasi totalité des clients vient de façon habituelle depuis de nombreuses années. Il nous est certes impossible de déterminer la part de chiffre d'affaires réalisée par les pratiquants dominicaux (elle est majoritaire), mais on retiendra malgré tout qu'une certaine population qui se déclare croyante considère la fréquentation d'une ou plusieurs kermesses comme un signe d'appartenance et de fidélité.

Au terme de ce premier inventaire, nous ferons deux commentaires. En premier lieu on soulignera l'individualisation des flux, telle qu'elle ressort de la pratique concrète. Que les paroisses catholiques soient alimentées financièrement par une masse de dons individuels non contrôlables (honoraires de messe et troncs) doit nous permettre de relativiser toute interprétation de l'activité paroissiale sous le prisme de l'exercice d'une autorité coercitive. Les paroisses, en fait, ne contrôlent pas aujourd'hui une part importante de leurs donateurs. Il faut cependant traiter à part, de ce point de vue, la pratique de la quête, qui est par excellence un geste sous contrôle social. Entre individualisation et socialisation, nous aurons à comprendre l'impact et le mode de fonctionnement des paroisses catholiques. Un deuxième élément nous est livré par l'observation : les pratiques de l'Église catholique se situent dans un temps long, et sous le mode de la transmission. L'archaïsme des modes de rémunération du clergé et d'alimentation des paroisses met en évidence le rôle essentiel des pratiques éducatives, et le souci du long terme qui se manifeste, probablement à l'insu même de ses initiateurs, dans les mises en œuvre concrètes de la vie religieuse.

18. Dans le Midi ou le Languedoc, le loto paroissial (quine) remplit les mêmes fonctions. 


\section{Casuel}

Le mot est bien connu des milieux ecclésiastiques, et son étymologie (de casus, « accident » en latin) révèle sa signification : le casuel est l'ensemble des ressources obtenues lors de cérémonies occasionnelles ( accidentelles ») : baptêmes, mariages, obsèques. Son importance a considérablement diminué au cours de ce siècle mais il constituait, il y a encore quarante ans, l'essentiel des ressources d'un curé.

À Paris, et dans les paroisses importantes comportant un curé et des vicaires, les ressources étaient réparties en fonction des cérémonies. Ainsi le curé se réservaitil les quêtes dominicales et le produit des troncs - par exemple -, le premier vicaire était attaché aux mariages, le second aux obsèques et le troisième aux baptêmes. À chacun revenait donc, comme unique ressource personnelle, la part d'offrandes versées par les fidèles, suivant un tarif plus ou moins officiel. On parlait ainsi des classes de mariage ou d'enterrements, car les cérémonies étaient hiérarchisées en fonction des ressources des personnes demandeuses. Dans ce système, le prêtre était directement tributaire de la fonction qui lui était attribuée (par l'évêque pour la cure, par le curé pour le vicaire) et de la capacité contributive des paroissiens, mais aussi de ses compétences personnelles. Avec Vatican II, les ressources du casuel ont été réintégrées dans le budget paroissial moyennant une simplification des tarifs (suppression des classes) et une garantie de revenus pour les clercs.

À l'heure actuelle, donc, le casuel est composé des dons effectués par les familles demandeuses de la cérémonie ${ }^{19}$ et qui sont le plus généralement payées par chèque, et des quêtes éventuelles qui ont lieu au cours de la cérémonie.

Le casuel est une ressource aléatoire, à la différence des troncs et quêtes qui constituent un flux régulier. Hormis le cas des églises situées près d'hôpitaux, qui sont assurées de nombreuses obsèques, la disparition des règles contraignantes de ressort géographique, l'urbanisation considérable et le développement des transports ont beaucoup favorisé la dissémination des cérémonies. Si la règle veut que le baptême et les obsèques aient lieu dans la paroisse du domicile et le mariage dans la paroisse de la fiancée, les dérogations sont très nombreuses. Ajoutons pour préciser l'observation que cette dissémination est surtout le fait des paroisses urbaines franciliennes, en raison des attaches provinciales d'une grande partie de la population (l'écart de coût d'organisation entre Paris et la province est aussi un facteur important).

Dans la pratique, la population concernée par le casuel est de deux catégories. Les paroissiens pratiquants constituent une partie minoritaire du « fonds de commerce ". Il convient de leur adjoindre toute une population qui ne pratique qu'au cours de ces cérémonies qui marquent les trois temps de l'existence : la

19. À Paris, en 2003, les obsèques sont « affichées » en moyenne entre 150 et 300 euros. 
vie, l'amour, la mort ${ }^{20}$. (Dans la troisième partie, une analyse à partir des dons nous renseigne sur ces pratiquants).

\section{Les cotisations pour la catéchèse}

Dans la définition canonique des tâches du pasteur de la paroisse (le curé), la catéchèse est inscrite dans les priorités. Souci éducatif, certes, mais bien plus tâche constitutive de la fonction curiale. Aussi les cotisations perçues à cette occasion enregistrent l'accord social entre la demande des croyants et la raison d'être de l'institution paroissiale.

L'inscription au catéchisme se fait à la rentrée scolaire, aux environs de la classe de CE2 (8-9 ans). Les procédures mises en œuvre pour promouvoir ces inscriptions amènent une foule de parents ayant de jeunes enfants, qui les inscrivent en même temps que les cours de musique ou de danse au Conservatoire municipal. Comme des «fournitures" sont prévues, de l'argent est versé. La ressemblance entre catéchisme et autres institutions éducatives pour les enfants est donc forte.

Des responsables (laïcs et prêtres) tentent en certains cas de s'opposer à cette perception très fonctionnaliste, en favorisant une implication directe (et quasi obligatoire) des parents dans l'acte catéchétique. Ainsi la catéchèse n'est pas un service analogue à l'école puisqu'il est annoncé comme service de la transmission familiale de la foi chrétienne. Les familles qui inscrivent leurs enfants au catéchisme sont en grande majorité des gens qui manifestent ainsi leur mode d'appartenance, peut-être des BME ayant un souci de la transmission plus marqué. Ils ne participent pas non plus à la messe dominicale.

Quoi qu'il en soit, la cotisation de catéchèse assure un revenu, certes non déterminant sur le plan financier mais stratégique dans le dispositif paroissial.

\section{Denier de l'ḱglise}

L'essentiel des ressources paroissiales demeure cependant le Denier de l'Église (DDE), encore souvent appelé Denier du Culte. Il est la version moderne du précepte de la dîme (= dixième) qui faisait l'objet d'un enseignement habituel de l'Église. La séparation de l'Église et de l'État, en 1905, qui a privé la première des ressources qui lui permettaient d'entretenir le clergé, a rendu ce précepte particulièrement nécessaire.

Il ne s'agit pas d'un impôt au sens propre du terme puisqu'il n'existe aucun système de contrainte dans l'Église qui en garantirait le recouvrement ; il ne s'agit pas non plus exactement d'un don à cause de son caractère régulier : celui qui verse

20. Parfois appelés «BME », pour Baptême, Mariage, Enterrement. Ils ont aussi le surnom de «chrétiens à roulettes ", qui ne viennent que voiturés : en poussette, en calèche, en corbillard. 
son DDE, bien qu'il soit libre d'y renoncer à tout moment, verse en général sa contribution tant que dure sa participation à la vie paroissiale, et particulièrement la fréquentation de la messe dominicale ${ }^{21}$. Il est actuellement la première source de financement des paroisses, représentant, en l'état actuel de nos recherches, de 40 à $60 \%$ du budget.

Le recouvrement du Denier de l'Église s'effectue au cours de deux campagnes menées auprès des fidèles et, d'une façon plus récente avec les affichages mis en place depuis une dizaine d'années, auprès du grand public. L'efficacité de ces appels de fonds demeure malgré tout liée à la population pratiquante. Un fichier est mis en place pour l'occasion, les fidèles reçoivent chez eux le tract les invitant à payer ; parfois des campagnes de proximité font partie de la stratégie de mobilisation des fidèles, relais des visites des prêtres auxquelles ceux-ci étaient autrefois tenus de se livrer une fois l'an. Les laïcs déposent alors dans les boîtes aux lettres ou donnent de la main à la main les tracts.

Pour les paroisses étudiées, le rapport financier du DDE est sans commune mesure avec la capacité contributive des cotisants. Il nous est possible, de ce fait, de comprendre le don fait à cette occasion sur un mode symbolique beaucoup plus que sur un plan fonctionnel. On retiendra en outre que les gros contributeurs sont également ceux qui consacrent le plus de leur temps à la paroisse ${ }^{22}$, au moins proportionnellement, et qu'il serait hasardeux d'envisager une substitution entre le temps dépensé et l'argent versé, comme s'il existait un système de compensation aux yeux des fidèles.

Le DDE représente habituellement l'essentiel des ressources paroissiales. Versé directement à la paroisse dans le cas de Paris ou à l'évêché pour la province, il est la ressource la plus régulière et la plus fiable en valeur des six paroisses étudiées (cf. tableau 1 ci-dessous). Seul le cas de Saint-Symphorien-Centre paraît atypique, avec des chiffres constamment inférieurs à $20 \%$. Il faut y lire la situation démographique très particulière du secteur ${ }^{23}$. Hormis ce point, les paroisses résidentielles s'assurent une ressource stable et majeure pour leur budget à travers le Denier de l'Église ${ }^{24}$.

21. Le développement des virements automatiques favorise cette dimension temporelle indéfinie du DDE. La paroisse St-Augustin de Bordeaux reçoit $80 \%$ de son DDE par virement mensuel ; la paroisse St-Jean-Baptiste de Grenelle, à Paris XVe 20 \% environ (chiffres 2004).

22. Indication obtenue par croisement des fichiers nominatifs: DDE et «membres de groupes paroissiaux» (ou équivalent).

23. Centre ville très paupérisé, avec trois églises.

24. Note méthodologique : compte tenu de l'hétérogénéité des rubriques comptables et des pratiques de Denier de l'Église, nous savons que Saint-Jacques prélève un quasi-Denier lorsque la paroisse propose une cotisation paroissiale et le Denier de l'Église officiel, reversé au diocèse. Le montant plus élevé qu'à Saint-Jules, par exemple, est dû : 1) à l'absence de quêtes au cours des messes dominicales et de troncs dans l'église ; 2) à l'histoire spécifique de Saint-Jacques, qui a longtemps prôné un système de Communauté de Base au militantisme fort. 
Tableau 1 : Pourcentage du Denier de l'Église dans les recettes globales de la paroisse ${ }^{25}$

\begin{tabular}{|c|c|c|c|c|c|}
\hline & \multicolumn{2}{|c|}{$\begin{array}{l}\text { DDE perçu et conservé } \\
\text { par la paroisse (Paris) }\end{array}$} & \multicolumn{3}{|c|}{$\begin{array}{l}\text { DDE perçu par le diocèse, ou par } \\
\text { la paroisse et reversé au diocèse (province }\end{array}$} \\
\hline Années & St-Jules & $\begin{array}{l}\text { Nin dar } \\
\text { Commerces }\end{array}$ & Ste-Thérèse ${ }^{1}$ & $\begin{array}{c}\text { Saint- } \\
\text { Symphorien- }^{\text {Centre }}{ }^{2}\end{array}$ & St-Jacques ${ }^{3}$ \\
\hline 1986 & 42,2 & nd & nd & nd & 54,4 \\
\hline 1987 & 41,7 & nd & nd & 16,2 & 55 \\
\hline 1988 & 43,3 & nd & nd & 15,9 & $48,2^{*}$ \\
\hline 1989 & 43,8 & nd & 38,7 & 13,4 & 46,9 \\
\hline 1990 & 43,9 & 33,8 & 33,9 & 15,5 & 42 \\
\hline 1991 & 42,8 & 33,2 & 33,8 & 18,5 & 49,5 \\
\hline 1992 & 41,1 & 34 & 31,6 & 13,8 & 46,6 \\
\hline
\end{tabular}

1. En rajoutant le DDE publié par le diocèse aux recettes ordinaires de la paroisse, qui ne le comptabilise pas.

2. En l'absence d'informations fiables, il s'agit de la subvention versée par le diocèse à la paroisse. À titre de comparaison, le DDE versé par les paroissiens au diocèse s'est élevé en 1992 à $12,3 \%$ des recettes. A partir de 1991, perte de la paroisse St-Paul.

3. Rubriques " cotisations" + "subvention du diocèse " (+ DDE de 86 à 88).

* Hors reversement au diocèse en 1988.

D'une façon plus précise, la paroisse de Saint-Jacques ${ }^{26}$ inventorie ses dons. 1485 items étaient inscrits dans le fichier paroissial en mars 1996 : étaient enregistrés aussi bien les inscriptions au catéchisme que la participation aux groupes liturgiques ou les versements aux Chantiers diocésains ${ }^{27}$. Le tableau ci-dessous $\mathrm{n}^{\mathrm{o}} 2$ résume les données majeures:

Tableau 2 : Répartition des dons comptabilisés par la paroisse Saint-Jacques ${ }^{28}$

\begin{tabular}{|c|c|c|c|c|c|c|c|c|c|}
\hline $\begin{array}{c}\text { DDE } \\
\text { seul }\end{array}$ & $\begin{array}{c}\text { DDE } \\
+ \text { caté }\end{array}$ & $\begin{array}{c}\text { DDE } \\
+ \text { autre } \\
\text { que caté }\end{array}$ & $\begin{array}{c}\text { DDE } \\
+ \text { caté } \\
+ \text { autre }\end{array}$ & $\begin{array}{c}\text { DDE } \\
+ \text { t cotis. } \\
\text { paroisse }\end{array}$ & $\begin{array}{c}\text { Cotis. } \\
\text { paroisse }\end{array}$ & $\begin{array}{c}\text { Cotis. } \\
\text { paroisse } \\
\text { + caté }\end{array}$ & $\begin{array}{c}\text { Cotis. } \\
\text { paroisse } \\
\text { + autre }\end{array}$ & $\begin{array}{c}\text { Caté } \\
\text { seul }\end{array}$ & $\begin{array}{c}\text { Caté } \\
+ \text { autre }\end{array}$ \\
\hline 274 & 9 & 80 & 7 & $\begin{array}{c}21 \\
\text { (dont } 13)^{*}\end{array}$ & $\begin{array}{c}616 \\
\text { (dont } 9)^{*}\end{array}$ & 0 & 39 & 243 & 60 \\
\hline
\end{tabular}

* «dont $\mathrm{N}$ » signifie que $\mathrm{N}$ cotisations paroissiales ont été versées par l'intermédiaire de l'œuvre Diocésaine, qui délivre des reçus fiscaux.

25. Le curé de Sainte-Blandine a laissé consulter les fichiers du DDE mais, paradoxalement, a été très réticent à fournir les comptes paroissiaux. D'où l'absence de données.

26. Ville de 60000 habitants dans une agglomération d'environ 600000.

27. Le fichier est anonyme. Sont répertoriés les dons et les inscriptions aux divers groupes paroissiaux en mars 1996.

28. Légende : DDE : Denier de l'Église ; Caté : cotisation à la catéchèse ; autre : argent versé pour une autre circonstance ; cotis. paroisse : cotisation paroissiale de Saint-Jacques, assimilable au DDE. 
Trois chiffres décrivent massivement l'imperméabilité des frontières culturelles dans la population paroissiale : 21 personnes allient «cotisation paroissiale » et "versement diocésain », 616 ne voient la paroisse qu'au travers de la pratique dominicale, 0 associent la vie paroissiale et la catéchèse ! Le tableau nous autorise à concevoir d'ores et déjà trois populations distinctes :

- le groupe des pratiquants dominicaux, pour qui la vie religieuse se traduit par une pratique cultuelle et, pour un petit nombre, par une participation aux activités paroissiales ;

- le groupe des fidèles qui ont une conscience institutionnelle forte : elle se traduit par un acte de reconnaissance de la dimension diocésaine. Ils appartiennent à l’Église au sens large;

- le groupe des usagers qui vient recevoir une identité catholique, pour laquelle ils ont versé leur écot (la cotisation au catéchisme, de 100 à $200 \mathrm{~F}$, ne constitue pas une adhésion de long terme ; elle est versée pour l'occasion et n'est pas relayée ni accompagnée d'une cotisation à l'institution).

Les données de Saint-Jacques, particulièrement accessibles et détaillées, sont corroborées par les observations que nous avons pu effectuer dans les trois paroisses parisiennes, qui comportent elles aussi une connotation de cotisation paroissiale (puisque le DDE est perçu par et au profit de la paroisse) ${ }^{29}$. Pour chacune d'elles, les taux de recouvrement sont certes plus élevés qu'à SaintJacques, mais toujours minoritaires : 27 noms sont communs aux fichiers « Denier de l'Église » et "catéchisme" à Sainte-Blandine (pour 418 DDE et 137 caté - soit 6,3 et 19,7 \% ${ }^{30}$ ), 21 à Saint-Jules (pour 1217 et 303 - soit 1,7 et 6,9\%), 12 à Notre-Dame-des-Commerces (pour 197 et 95 - soit 6,1 et $12,6 \%$ ) $^{31}$.

Un dernier exemple d'analyse comptable va nous permettre de poser une question sur les croyants non (ou très peu) pratiquants, ceux que nous avons appelé les «BME ». Qui sont-ils, et surtout, que viennent-ils demander à l’Église catholique?

Les BME contribuent au financement de la paroisse même si les sommes ainsi collectées ne représentent plus qu'une faible part des ressources dans les budgets. Le tableau $n^{\circ} 3$ ci-après nous indique les spécificités de chaque paroisse, et leur

29. L'absence de cotisation paroissiale à Sainte-Thérèse de Petilac ne permet pas de tirer d'enseignement de l'analyse des fichiers.

30. Le point de comparaison, donateurs/DDE ou donateurs/caté, dépend de ce que l'on veut montrer. À Sainte-Blandine, 19,7 \% correspond presque exactement à la population « jeunes » de l'arrondissement (moins de 18 ans). C'est le seul cas.

31. Si le taux de recoupement Catéchèse-DDE (ou cotisation paroissiale) est faible, il faut en incriminer, outre l'hétérogénéité des groupes sociaux, la différence d’âge. On peut émettre l'hypothèse que seul l'âge élevé des donateurs au DDE les exclut de facto du groupe catéchisme ; mais qu'en est-il des parents du catéchisme ? Il est probable que le don aux institutions est le fait des gens ayant dépassé l'âge de l'enfantement (25-45 ans), mais la corrélation avec le niveau des ressources disponibles reste incertaine. 
degré de lien avec ces pratiquants occasionnels : les chiffres montrent clairement que les paroisses de l'enquête vivent très différemment la place des BME dans leur activité.

Malgré les correctifs indispensables - à Saint-Jacques, les quêtes dominicales étant quasi inexistantes, la part des autres recettes en est arithmétiquement augmentée -, on perçoit combien les physionomies financières paroissiales sont fonction de la population des fidèles :

Tableau 3 : Part du casuel dans les recettes paroissiales ordinaires (en \%)

\begin{tabular}{|l|r|r|r|r|r|r|r|r|r|r|}
\hline & 1986 & 1987 & 1988 & 1989 & 1990 & 1991 & 1992 & 1993 & 1994 & 1995 \\
\hline Ste-Thérèse ${ }^{1}$ & - & 18,5 & 12,2 & 11,3 & 7,4 & 8 & 10,8 & 11,3 & 11,8 & 16,7 \\
\hline St-Jacques & 17,8 & 17,3 & 19,7 & 21,8 & 21,6 & 26,2 & 25,2 & 25 & 23,3 & 27,3 \\
\hline $\begin{array}{l}\text { St-Symphorien- } \\
\text { Centre }\end{array}$ & - & 8,8 & 9,2 & 9,8 & 8,2 & $11,3^{2}$ & 13 & 11 & 15,2 & 13 \\
\hline $\begin{array}{l}\text { S.-Symph., } \\
\text { dont Offrandes }\end{array}$ & - & 6 & 5,8 & 6,4 & 4,8 & 82 & 8,2 & 7,2 & 9,7 & 9,1 \\
\hline $\begin{array}{l}\text { St-Jules } \\
\begin{array}{l}\text { ND des } \\
\text { Commerces }\end{array}\end{array}$ & 10,4 & 9,5 & 8,2 & 6,8 & 7,6 & 8 & 8,6 & - & - & - \\
\hline
\end{tabular}

1. Après réintégration du DDE dans le budget.

2. Au 30 juin, l'église St-Paul est détachée de l'ensemble.

3. Y compris les Intentions de Messe.

Un examen plus attentif des pratiques comparées permettra de circonscrire plus étroitement les particularités propres à chaque paroisse et de repérer les invariants. Nous nous proposons, à cet effet, de reporter dans le détail les différents chiffres de cérémonies ainsi que les tarifs pratiqués par les paroisses.

Tableau 4 : Casuel détaillé de la paroisse Saint-Jules

\begin{tabular}{|c|c|c|c|c|c|c|c|c|c|c|c|c|c|c|c|}
\hline & \multicolumn{3}{|c|}{1988} & \multicolumn{3}{|c|}{1989} & \multicolumn{3}{|c|}{1990} & \multicolumn{3}{|c|}{1991} & \multicolumn{3}{|c|}{1992} \\
\hline & $\mathrm{Mrg}$ & Obs & Bpt & $\mathrm{Mrg}$ & Obs & Bpt & Mrg & Obs & Bpt & Mrg & Obs & Bpt & $\mathrm{Mrg}$ & Obs & Bpt \\
\hline Nombre & 37 & 206 & 148 & 55 & 156 & 157 & 61 & 181 & 136 & 56 & 170 & 154 & 62 & 203 & 169 \\
\hline $\begin{array}{c}\text { Casuel } \\
\text { global (KF) }\end{array}$ & 46,6 & 206 & 27 & 60 & 163 & 27,6 & 82,5 & 180 & 25,7 & 83 & 194 & 32,2 & 87,3 & 231 & 34,9 \\
\hline Don moyen $(F)$ & 1260 & 999 & 182 & 1090 & 1044 & 175 & 1352 & 997 & 189 & 1482 & 1139 & 209 & 1408 & 1137 & 207 \\
\hline $\mathrm{Mrg} / \mathrm{bpt}$ & & 6,92 & & & 6,23 & & & 7,2 & & & 7,1 & & & 6,8 & \\
\hline Mrg/obs. & & 1,26 & & & 1,04 & & & 1,37 & & & 1,3 & & & 1,24 & \\
\hline Obs/bpt & & 5,48 & & & 5,96 & & & 5,28 & & & 5,45 & & & 5,49 & \\
\hline
\end{tabular}


Remarques :

La baisse brutale et sans lendemain du don aux mariages en 1989 est peut-être tributaire d'une erreur de comptage, d'un oubli de quête ou autre erreur d'imputation ; il est difficile de statuer dessus.

La hausse importante pour les obsèques en 1991 s'explique vraisemblablement par l'augmentation du tarif indicatif fourni aux familles lorsqu'elles se présentent à la paroisse pour des obsèques.

À partir des chiffres fournis par la paroisse (tableau 4), nous calculons un don moyen fait à l'occasion de chaque type de cérémonie. Toujours dans le dessein de remédier à l'hétérogénéité des cérémonies dans leur rendement financier, nous avons recherché un rapport constant entre les cérémonies; les résultats sont regroupés dans les trois dernières lignes du tableau. Nous constatons de fait que le versement moyen des fidèles, à prescriptions égales de la part de la paroisse, s'inscrit dans une extraordinaire homogénéité, indiquant ainsi un comportement de don ancré dans les mentalités. En moyenne, un mariage rapporte 1,24 fois plus qu'un convoi, 6,85 fois plus qu'un baptême.

Il nous est dès lors possible de calculer une unité de cérémonie (en ce qui concerne le taux de contribution), l'unité-baptême : un mariage vaut 6,85 baptêmes, un enterrement vaut 5,53 baptêmes. Sous l'hypothèse, maintenant, d'une proportionnalité constante dans les paroisses de l'enquête, nous pouvons additionner les cérémonies en "unités-baptême " de façon à rendre comparables les comportements financiers des fidèles dans les différentes paroisses. D'où le tableau suivant $\left(\mathrm{n}^{\circ} 5\right)$, établi en termes de moyennes constatées sur plusieurs années à partir des indications reçues au cours de l'exploration de terrain :

Tableau 5 : Capacité contributive des cérémonies célébrées (nombre estimé en 1990)

\begin{tabular}{|l|c|c|c|c|c|c|}
\hline & Baptêmes & $\begin{array}{c}\text { Mariages } \\
\text { (équiv. } \\
\text { Bapt.) }\end{array}$ & $\begin{array}{c}\text { Obsèques } \\
\text { (équiv. } \\
\text { Bapt.) }\end{array}$ & $\begin{array}{c}\text { Total } \\
\text { en équiv. } \\
\text { bapt. }\end{array}$ & $\begin{array}{c}\text { Casuel } \\
1990 \\
\text { en KF }\end{array}$ & $\begin{array}{c}\text { Casuel/ } \\
\text { cérém. } \\
\text { en F }\end{array}$ \\
\hline Saint-Jules* & 136 & $\begin{array}{c}61 \\
(418)\end{array}$ & $\begin{array}{c}181 \\
(1001)\end{array}$ & 1555 & 289 & 186 \\
\hline Sainte-Thérèse & 80 & $\begin{array}{c}30 \\
(206)\end{array}$ & $\begin{array}{c}50 \\
(276)\end{array}$ & 562 & 22,7 & 40,4 \\
\hline $\begin{array}{l}\text { St-Symph.- } \\
\text { Centre }\end{array}$ & 25 & $\begin{array}{c}18 \\
(123)\end{array}$ & $\begin{array}{c}40 \\
(221)\end{array}$ & 369 & 53,3 & 144 \\
\hline Saint-Jacques & 200 & $\begin{array}{c}100 \\
(685)\end{array}$ & $\begin{array}{c}250 \\
(1382)\end{array}$ & 2267 & 158 & 70 \\
\hline
\end{tabular}

* Chiffres exacts pour Saint-Jules.

Nous observons en premier lieu les écarts des capacités contributives entre les paroisses : de 1 à 4,5. Une telle dispersion interroge tout de suite notre hypothèse 
de base, à savoir les équivalences de proportion pour les dons aux cérémonies. La paroisse Sainte-Thérèse a un taux de recouvrement infiniment plus faible que Saint-Jacques où l'on ne donne aucune indication, ou Saint-Symphorien-Centre dont l'exiguiité sociale est relativement voisine. Saint-Jules, la paroisse la plus prospère, n'est toutefois pas tellement plus contributive que Saint-SymphorienCentre.

L'écart de contribution que nous avons relevé entre les baptêmes, d'un côté, et les mariages et enterrements s'explique aisément par le facteur «nombre de participants » : qui a observé ou participé à un baptême se rend compte qu'il concerne le seul noyau familial proche, et que le nombre de personnes présentes ne peut guère contribuer financièrement au même niveau qu'un mariage. Celuici, en revanche, convoque deux familles et leurs alliés, ce qui permet déjà de doubler au moins la contribution. Quant aux obsèques, si bien des services funèbres dans les paroisses parisiennes ne rassemblent qu'un petit nombre de personnes (la famille est fréquemment dispersée), elles sont encore le lieu où des personnes extérieures à la famille peuvent manifester leur soutien : voisins, connaissances éloignées, etc. Dès lors que la cérémonie est connue deux ou trois jours à l'avance, il est justifié que l'on y participe nonobstant les activités professionnelles.

Être BME, c'est venir demander à l'Église catholique, quelles que soient les croyances que l'on professe, un ensemble de rites qui ponctuent les grandes étapes de la vie. L'argent que l'on verse à cette occasion signifie le prix auquel on estime de tels passages, et il faut à cet égard dépasser les apparences : malgré les rendements plus élevés des mariages, les paroisses ont mis en place une structure de prix qui fait de la mort l'événement le plus onéreux. Proportionnellement, les gens donnent beaucoup plus aux obsèques qu'aux mariages, et la politique tarifaire en tient compte.

Prenons l'exemple de Sainte-Thérèse: selon les indications du curé, 100 personnes au mariage rapportent 40,4 $\mathrm{F} \times 6,85$ (taux de conversion en unitébaptême $)=276,7 \mathrm{~F}$, soit $2,8 \mathrm{~F}$ par personne, alors que 50 personnes aux obsèques donnent : 40,4 F $\times 5,53=223,4 \mathrm{~F}$, soit $4,5 \mathrm{~F}$ ! La hiérarchie réelle (encore une fois sur la base d'estimations et en attente de confirmation chiffrée précise) n'est donc pas celle observée par l'Église mais bien : a) la mort, b) la formation de la famille, c) la naissance. Dieu est plus sollicité - ou bien on attend plus de lui - en cas de décès qu'en cas de naissance. Risquons alors une interprétation.

Les trois passages majeurs de la vie sont trois passages décisifs au cours desquels se décide la vie. À ce moment précis de l'aventure humaine se joue la question de la survie : c'est l'évidence même pour la mort ; la mesure en paraît moindre pour le mariage parce que le lien conjugal a été partiellement découplé de la fécondité, encore moins pour le baptême dans la mesure ou la régression importante de la mortalité infantile a rendu l'intervention divine moins nécessaire. 
En ce qui concerne les baptêmes, les réunions de préparation qui ont lieu dans les paroisses révèlent souvent une peur latente chez les parents (la mère surtout, mais aussi la grand-mère) "qu'il arrive quelque chose ". La hiérarchie des dons correspond alors non pas au degré vital de chaque étape, mais au degré de contrôle que l'homme peut exercer sur ce passage. Il est nul en ce qui concerne l'au-delà, c'est la raison d'un don élevé ; il est plus faible pour la naissance en raison des progrès de l'hygiène publique ainsi que pour la fécondité, mieux contrôlée par la médecine. Recourir aux services offerts par l'Église catholique s'impose donc lorsqu'il s'agit d'exorciser le risque couru par les gens. Selon une expression triviale, il s'agit de "mettre Dieu dans sa poche ", de prolonger le désir de vivre là où leur âme n'est pas le maître, en le confiant au Maître absolu de ce lieu géographiquement inexistant dont l'Église est supposée la gardienne.

\section{Dépenses : la gestion paroissiale}

Les paroisses dépensent l'argent qui leur est versé de façon classique pour toute activité institutionnelle. Nous verrons plus loin en détail les masses représentées pour Saint-Jules (Paris) et Saint-Jacques (province). Ici, contentons-nous de lister les emplois possibles et habituellement constatés.

À tout seigneur, tout honneur : le personnel représente le plus gros poste de dépense de toutes les paroisses étudiées, si, toutefois, on prend soin de réintégrer les émoluments des clercs dans les paroisses de province où ils sont payés par le diocèse. Parfois, les comptes ne permettent pas de distinguer du personnel laïque (administratif, d'entretien, voire pastoral) : en effet, les prêtres «coûtent » bien moins cher que les laïcs.

Les frais entraînés par le parc immobilier constituent en général une grosse source de dépenses, souvent la deuxième en importance. Même lorsque les paroisses sont sous le régime de l'affectation des biens (église et presbytère), elles doivent entretenir le patrimoine au-delà des mises en sécurité ou du gros-œuvre assumé par les communes. Peintures, électricité, sonorisation, mobilier, etc. sont à leur charge $^{32}$. À cela s'ajoutent les divers frais de matériel de bureau, fournitures diverses comme l'eau, l'électricité, etc.

La solidarité avec le diocèse comprend à la fois le financement du gouvernement diocésain (services divers, évêché) et la redistribution en faveur des paroisses sans moyens. Il faut bien entendu neutraliser les différences de prélèvements d'un diocèse à l'autre pour entrer dans une comparaison inter-diocésaine, et procéder

32. Telle grosse paroisse de Paris, disposant de $3000 \mathrm{~m}^{2}$ de locaux - dont quelques centaines seulement sous le régime affectataire - doit assurer un entretien de 600 euros par $\mathrm{m}^{2}$ (peintures, plomberie, électricité, mise aux normes). En prenant une hypothèse économe d'un amortissement sur 15 ans, on arrive à un budget d'entretien qui devrait être de 120000 euros par an; soit plus du quart du DDE en 2004. 
à des retraitements sur le DDE et les frais de personnel. L'omission de cette précaution rend les chiffres publiés inutilisables.

Enfin, avec le reliquat, les paroisses assurent parfois des financements d'actions de solidarité (hors cas des activités de jeunesse, qui sont à usage interne bien souvent). Il s'agit par exemple de subventions accordées à des organismes extérieurs à la paroisse (œuvres de jumelage, telle ou telle paroisse ou aumônerie pour une action ponctuelle, etc.). Mais, la plupart du temps, les paroisses se contentent d'organiser des quêtes dont le produit ira directement au destinataire. C'est ainsi que nous avons pu mettre en évidence, grâce aux chiffres fournis par le conseil économique de St-Jules, que les quêtes organisées au profit de l'extérieur étaient, de 1986 à 1993, équivalentes aux quêtes faites au profit de la paroisse : pour un franc donné à la paroisse, un franc a été donné à l'extérieur ${ }^{33}$ !

L'excédent éventuel de gestion (pour les paroisses importantes) est capitalisé en vue des gros investissements ultérieurs. Hormis la paroisse Saint-Jules (la plus riche de notre échantillon), la contrainte budgétaire bannit toute thésaurisation massive, et l'existence de conseils de gestion composés de personnalités diverses préserve des dépenses somptuaires - ce qui n'exclut pas, bien évidemment, la possibilité d'une gestion hasardeuse à telle ou telle occasion lorsque les régulations ne fonctionnent plus.

\section{Deux budgets comparés}

Saint-Jacques et Saint-Jules drainent toutes les deux des foules, comme le remarque le $\mathrm{CP}^{34}$ de Grosport lorsqu'il comptabilise un millier de personnes passant dans la semaine pour cause d'obsèques - la population du casuel compensant la plus forte participation aux messes dominicales de Saint-Jules. Les deux paroisses sont comparables, grosso modo, en termes d'impact sur une population, mais leurs puissances financières sont loin de l'être.

Étonnons-nous, d'abord, des similitudes de comportement, qui concernent l'entraide et la gestion courante. Cette dernière est aussi rigoureusement tenue dans l'une et l'autre paroisse, comme l'indiquent les pourcentages de part et d'autre. En additionnant les moyennes sur sept ans des TFSE et FdG, qui représentent l'ensemble des dépenses relativement nécessaires pour assurer la vie courante - chauffage, électricité, assurances, petit matériel, fournitures de culte, etc. -, on obtient pour Saint-Jules $22 \%$ et Saint-Jacques $20 \%$; la première jouit seulement d'une liberté plus grande dans ses contraintes, et elle s'est autorisé $10 \%$ de dépenses de plus que Saint-Jacques, ce qui est signe de sagesse.

33. À titre d'exemple, montants bruts des collectes : 1985 : $444 \mathrm{KF}$; 1991 : $621 \mathrm{KF}$. Quêtes dominicales : 1985 : $459 \mathrm{KF}$; 1991 : $719 \mathrm{KF}$. Il est courant, dans les présentations financières, d'utiliser l'abréviation « KF » pour parler des milliers de francs. Ainsi : $300 \mathrm{KF}=300000 \mathrm{~F}$.

34. Conseil pastoral. 
86 - ARCHIVES DE SCIENCES SOCIALES DES RELIGIONS
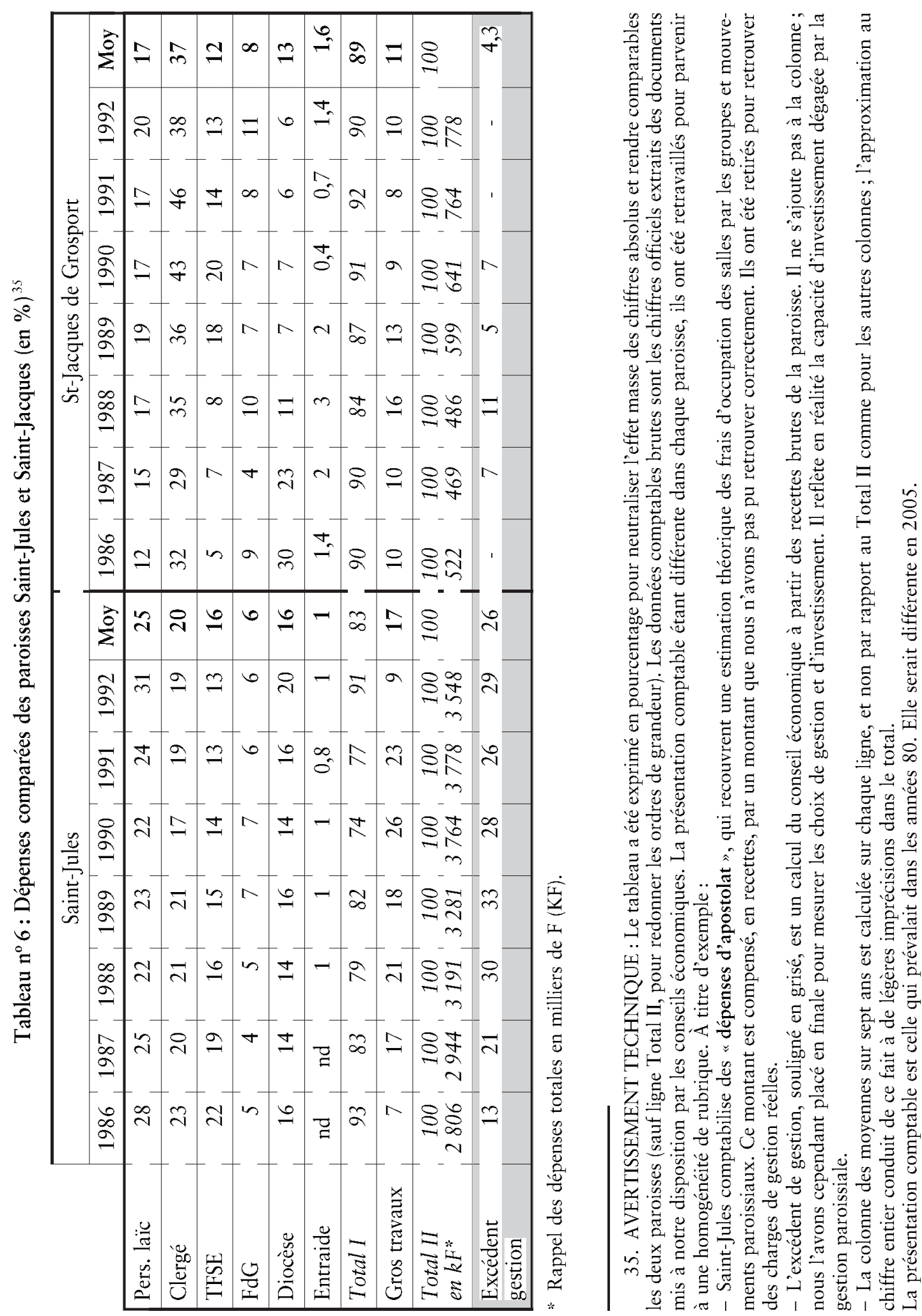
L'entraide ${ }^{36}$ est apparemment équivalente dans les deux paroisses ; on aurait pu augurer d'un effort supérieur à Saint-Jacques compte tenu de son histoire. En réalité, la contrainte de gestion est telle pour la paroisse que l'effort d'entraide est significativement plus élevé qu'à Paris : si nous la rapportons à l'excédent de gestion, il ressort que Saint-Jules arbitre très nettement en faveur de l'investissement immobilier au détriment de l'entraide. Pour un excédent moyen, sur la période, de $26 \%$ à Paris et de 4,3\% à Grosport, l'effort assumé par cette dernière est proportionnellement près de 8 fois supérieur.

Les différences de gestion apparaissent plus nettement sur la rémunération du personnel et l'excédent de gestion.

Côté personnel, la répartition s'effectue de façon significativement plus cléricale à Grosport qu'à Paris. Sur sept ans, nous découvrons que Saint-Jules consacre en moyenne respectivement $25 \%$ et $20 \%$ aux laïcs et aux prêtres là où SaintJacques offre $17 \%$ et $37 \%$ (orientation corroborée par les entretiens : les prêtres de Grosport jouissent d'une rémunération plus élevée dans cette paroisse que les autres prêtres du diocèse). Indépendamment de ces choix de rémunération, les chiffres manifestent que le rapport à l'argent des clercs ne s'établit pas sur des bases semblables dans les deux paroisses. En constatant en effet que les prêtres sont les principaux responsables des rentrées financières, - et s'ils ne le font pas directement, leur présence est indispensable puisqu'ils délivrent les actes cultuels au cœur de l'activité paroissiale -, deux interprétations sont possibles. Ou bien, en adoptant une vue de gestionnaire d'entreprise, le clergé de Saint-Jacques est moins «productif». Ce qui peut encore s'énoncer en affirmant que l'activité paroissiale de Saint-Jacques est largement tributaire du clergé. Ou bien on dira que la paroisse Saint-Jacques n'est pas tant tournée vers l'entretien du matériel (immeubles, institutions, etc.), auquel elle concède ce qui est juste (d'où la similitude de gestion avec Saint-Jules), que vers une vie plus immatérielle. Les prestations requises des paroissiens ne sont pas financières, ce qui laisse supposer un autre ordre de prestations. Si tel est le cas, il reste à en trouver le "marqueur » qui les rendra visibles.

L'excédent de gestion quasi inexistant de Saint-Jacques vient corroborer cette dernière interprétation. Avec une moyenne sur sept ans de 4,3\%, la paroisse se distingue par son délicat équilibre financier, tandis que Saint-Jules donne le visage d'une entreprise très rentable : $26 \%$ d'excédent moyen! Nuançons cet apparent écart, afin de rendre comparable ce qui l'est. Il est possible d'effectuer une simulation sur les budgets.

Supposons que les troncs et les quêtes de Saint-Jacques suivent le modèle courant. Corrigeons le budget de 1989 (celui dont l'excédent de gestion s'approche le plus de la moyenne) en rétablissant fictivement des quêtes : nous avons établi

36. Sous les réserves posées dans l'avertissement technique. 
que les paroissiens donnent en moyenne $2 \mathrm{~F}$ à la quête dominicale, contre 5 à $7 \mathrm{~F}$ ailleurs. Nous pouvons sans grand risque d'erreur choisir le chiffre de $5 \mathrm{~F}$ par personne, c'est-à-dire multiplier les recettes des quêtes par 2,5. Par ailleurs, compte tenu de l'emplacement géographique de l'église, la consommation de cierges devrait être plutôt élevée. Sachant d'une part que les cierges en 1996 ont rapporté plus de $2000 \mathrm{~F}$ par mois dès leur installation, et que les troncs laissés en semaine dans l'église ont rapporté en 19931500 F mensuellement sans qu'il $\mathrm{y}$ ait de cierges à l'époque, on peut raisonnablement tabler sur $1500 \mathrm{~F}$ pour 1989 (estimation conservatrice, mais qui tient compte de l'inflation sur quatre ans). Au total, le budget corrigé de Saint-Jacques donnerait $350 \mathrm{KF}$ pour les quêtes (au lieu de $143 \mathrm{KF}$ ) et $18 \mathrm{KF}$ pour les cierges, qui s'ajouteraient à l'excédent de gestion (35 KF) pour donner un excédent théorique de : $398 \mathrm{KF}$, soit $62 \%$ des dépenses totales.

L'excédent de gestion s'apparenterait alors plus nettement à celui dégagé par Saint-Jules. Entre les $33 \%$ de cette dernière en 1989 et les $62 \%$ théoriques de Saint-Jacques, qui seraient probablement ramenés à une valeur voisine de sa consœur parisienne (surcroît de dépenses pour l'immobilier et la gestion), nous percevons bien les choix importants faits par l'équipe dirigeante de la paroisse de province, et le prix à payer. La marge de gestion du CE (conseil économique) est en réalité entièrement confisquée par les instances politiques de la paroisse, qui réduisent ainsi le conseil à effectuer un travail sous forte contrainte.

Saint-Jules, au contraire, dispose de réserves étonnantes. La constitution de provisions pour gros travaux ou investissement, comme ce fut le cas en 1991 et 1992 en vue d'une opération immobilière (1,2 MF environ $)^{37}$ donne un réel pouvoir au CE, qui peut ensuite, s'il le souhaite, orienter les choix pastoraux par la mise en évidence de critères de gestion. À vrai dire, la puissance financière n'est pas à elle seule un facteur de pouvoir. Un jeu subtil s'établit entre les instances dirigeantes (au sens large) de la paroisse à partir du moment où la marge de manœuvre financière est publiée, autorisant ainsi les rêves et les possibilités de dépenses : les négociations s'établissent sur la concrétisation de ces rêves.

La différence de gestion manifestée par les comptes de Saint-Jacques et SaintJules porte vraisemblablement sur l'imaginaire qui meut les instances dirigeantes des deux paroisses : pour l'une, il n'y a aucune crainte à ce que le budget paroissial rassemble les multiples dons individuels pour affecter les ressources; pour l'autre, il convient au contraire de laisser les fidèles libres d'affecter individuellement leurs ressources où ils le veulent.

37. L'information n'apparaît pas dans le compte d'exploitation, puisqu'il s'agit de stocks, sinon en suivant sur très long terme la constitution des provisions. Ici, l'information provient des comptes-rendus du CE. 


\section{Les catholiques et le don d'argent}

Entre commerce et don, il faut choisir : les intentions de messe, les cierges, les cérémonies (casuel) sont-elles " vendues ", ou bien s'agit-il de dons ? Formellement, il s'agit de dons, bien que l'automaticité du paiement accrédite la vraisemblance de la première hypothèse. Il ne lui manque qu'un refus manifeste de les fournir gratuitement, de la part des paroisses, pour devenir certaine. Les paroisses catholiques donnent certes l'impression à l'observateur extérieur qu'elles font commerce des faveurs divines; mais, à notre connaissance, personne ne se fait renvoyer parce qu'il ne dispose pas de la somme nécessaire. C'est pourquoi nous penchons plutôt en faveur de la seconde interprétation, quand bien même, dans l'imaginaire des pratiquants (et spécialement des occasionnels), la seconde serait plus présente. L'affaire est tout aussi valide pour les participants de la catéchèse, puisqu'il s'agit d'une cotisation (ou participation aux frais) : le refus par la paroisse d'accueillir un enfant lorsque les frais n'ont pas été payés n'a pas été porté à notre connaissance. Du point de vue paroissial, il ne peut être question d'un commerce; du point de vue des parents, l'interprétation est sans doute plus floue.

En tout état de cause, les travaux menés sur ces paroisses (et corroborés pour d'autres périodes sur d'autres terrains) dessinent un panorama en matière de dons. D'un côté se trouvent les différents groupes de fidèles qui viennent pour une demande ponctuelle, circonstanciée, limitée dans le temps; de l'autre, les fidèles « illimités ", c'est-à-dire qui abondent de façon récurrente aux budgets des paroisses. Pour l'essentiel, ce sont les cotisants au DDE, qui sont également pratiquants le dimanche (et donc, remplissent les quêtes dominicales).

Les premiers participent de près ou de loin à l'activité déployée par le clergé. L'aspect limité de leur demande constitue leur caractéristique commune. L'examen minutieux des pratiques financières, complété par des entretiens, nous autorisent à estimer qu'ils sont répartis en quatre groupes : les individuels, qui ne pratiquent que les cierges; les BME (le casuel); les parents de l'initiation (catéchèse); les fidèles des défunts. Ces catégories se recouvrent partiellement, il faudrait de gros moyens d'enquête pour l'établir dans le détail. Tous les fidèles définis selon ces caractéristiques mesurent leur fidélité, et l'acte de paiement qui s'ensuit est également mesuré. En cela ils se distinguent du dernier groupe de fidèles, les pratiquants messalisants.

Formant la partie visible permanente de la vie catholique, les messalisants sont les plus gros contributeurs de la vie paroissiale. Ils assurent par leur fidélité active à la fois la permanence du don dans la vie sociale, et l'animation régulière du groupe catholique. Leurs pratiques de don sont à l'image de leur investissement humain : ils donnent sans compter (quête dominicale), ils n'interrompent pas leur don, ils vont parfois jusqu'à inscrire leur fidélité dans leur budget mensuel (prélèvement du DDE). Les fidèles pratiquants forment donc un groupe à horizon de fidélité illimitée. S'ils recourent aux services auxquels les autres fidèles font 
appel : intentions de messe, catéchèse, cérémonie sacramentelle, ils le font comme un complément naturel de leur activité religieuse ordinaire. Leur comportement a un impact significatif sur le paysage de la générosité en France, selon le Baromètre de la générosité.

La Fondation de France a lancé depuis plus de dix ans des enquêtes approfondies sur le don en France. Ces enquêtes ont partiellement recoupé des études européennes. Elles décrivent un panorama du don à la fois très fin et très complet ${ }^{38}$, portant sur les motivations, sur les pratiques, sur la répartition géographique, etc.

L'étude sur la générosité des Français donne quelques chiffres significatifs ${ }^{39}$. Le travail ayant été réalisé à partir des données fiscales, d'une part, et de sondages auprès de la population, d'autre part, cette publication (la huitième consécutive) est un baromètre fiable. Les auteurs avancent ainsi une somme comprise entre 1,6 et 1,9 milliards d'euros, (10,5 - 12, 5 milliards de francs) pour 2002. La répartition des dons entre les foyers imposables - qui peuvent bénéficier d'un abattement fiscal - et foyers non imposables, pour qui la déclaration n'est pas «fiscalement utile " montre que la générosité n'est pas liée à un intérêt bien compris. En ce sens, l'étude confirme le côté non utilitaire du don, ou plutôt la gratuité possible du don : à peine plus d'un foyer imposable sur cinq $(21,4 \%$ en 2001) déclare un don. Faut-il pour autant tabler sur une volonté de discrétion et de gratuité ? Un sondage Sofres pour l'UNOGEP ${ }^{40}$ de 2002 indique que, parmi les hauts revenus, moins de $20 \%$ des personnes passeraient sous silence leur don. Les auteurs en concluent que "plus de six Français sur dix indiquent ne jamais donner d'argent ». L'âge est une variable importante et «cela paraît être la conséquence de l'augmentation des moyens disponibles, de par l'évolution des revenus et de par la diminution des charges ».

La variable religieuse n'est donc pas anodine dans la formation d'une conscience sur le don. Le Baromètre de la générosité $2002^{41}$ indique que :

- si $29 \%$ des Français ont fait un don en mars-avril 2002, $67 \%$ de ces donateurs étaient catholiques ;

- et $41 \%$ des catholiques pratiquants ont fait un don (en mars-avril 2002) contre seulement $22 \%$ des catholiques non pratiquants.

Les auteurs de l'enquête ne cherchent pas à rapporter ces chiffres à la pratique dominicale (environ 10 \% en France). Si on applique les coefficients, on s'aperçoit

38. Ces études n'étaient pas disponibles sur la période qui a servi de base à notre enquête. De ce fait, les rapprochements ne valent qu'à titre d'hypothèses à forte dose de vraisemblance. Il faudrait entreprendre des études sur les comptes des paroisses et diocèses pour 1995-2003.

39. Sylvie Malsan ; Jacques Malet, La générosité des Français. Étude sur les dons déclarés sur 10 années (mars 1994 à mars 2003), Paris, Fondation de France, 2003.

40. Sondage Sofres effectué en avril 2002 pour l'Union Nationale des Organismes faisant appel à la Générosité Publique.

41. Baromètre de la générosité en France. Enquête réalisée par la Sofres pour la Fondation de France, Paris, octobre 2002. 
que les catholiques pratiquants pourraient représenter : $0,67 \times 0,41=27,5 \%$ des Français donateurs, soit près de trois fois leur représentation démographique !

Tous les indicateurs convergent donc dans cette direction : la pratique religieuse est un facteur déterminant du passage au don, c'est-à-dire du détournement du circuit monétaire tel que la société l'organise. C'est donc dans ce contexte où les dons semblent commandés par une appartenance religieuse - pour ce que nous en savons, majoritairement catholique - que nous devons replacer le fonctionnement et la place des paroisses catholiques dans l'économie générale de la circulation monétaire en France.

\section{Conclusion}

La paroisse catholique française est une réalité complexe. L'hétérogénéité des fidèles qui recourent à ces services en est à l'origine. Pour les uns, les groupes à horizon de fidélité limitée, il s'agira sans doute de services analogues aux services publics, accessibles à tous selon les besoins exprimés par chacun. Pour les autres, ceux du noyau dur, il s'agit plutôt d'un espace social au sein duquel une vie de croyants est poursuivie sur le long terme. Cette vie comprend différentes modalités (sacrements, groupe de réflexions du service social, ...) qu'unifie la fréquentation de la messe dominicale. Les fidèles messalisants fournissent encadrement nécessaire à l'animation de l'ensemble, mais la place du clergé demeure décisive dans le dispositif, particulièrement pour ce qui concerne la satisfaction de demandes ponctuelles.

L'approche par la comptabilité confirme cette hétérogénéité. Elle montre en revanche l'unification des pratiques catholiques, au moins en ce qui concerne la perception des recettes. À ce titre, on peut légitimement concevoir que les paroisses sont analogues aux succursales des entreprises commerciales. La convergence de la gestion (quelle est son origine ?) atteste de l'existence d'une politique de gestion du religieux au niveau de l’Église catholique en France.

Nicolas de BREMOND D'ARS

Centre d'Études Interdisciplinaires des Faits Religieux - EHESS-CNRS 
92 - ARCHIVES DE SCIENCES SOCIALES DES RELIGIONS

Mots-clés : argent, catholicisme, don, paroisse

\section{Résumé}

Les règles de comptabilité qui s'appliquent aux paroisses catholiques rendent lisibles les flux d'argent qui y transitent. Les méthodes d'analyse comptable et financière offrent le moyen de décrypter l'univers des pratiques religieuses catholiques en France: les recettes proviennent de divers actes rituels, et les dépenses obéissent à des impératifs techniques aussi bien qu'à des imaginaires religieux. Il apparaît de façon singulière que les catholiques ayant recours aux services des paroisses appartiennent à des sousgroupes distincts, contigus mais hétérogènes les uns aux autres. Si la forme du don est la même pour tous, en raison de la réglementation, les pratiquants réguliers apparaissent proportionnellement, selon les données fiscales, comme les plus généreux en France. Pour les autres catholiques, on demeurera indécis quant à savoir s'il s'agit d'un commerce de "biens de salut " ou d'un don.

\section{Abstract}

Accounting rules for catholic parishes give readability to the money flows which pass through. The cost-accounting methods present to the sociologist the possibility of elucidate the catholic univers of rituality in France: receipts come in regarding different ritual acts, and spendings follow technical imperatives as well as religious imaginaries. It singularly appears that catholic believers having recourse to the parish services belong to distinct subcultures, which are contiguous but heterogenous. The accounting transaction form is similar - a donation-, besause of the accounting and fiscal rules, but the regularly catholic churghgors appears to be, according to the tax-administration's informations, the most generous givers in France. We stay prudently behind for less catholic practiquants, hesitating between real donation or buying a religious good.

\section{Resumen}

Las reglas de contabilidad aplicables a las parroquias catolicas permiten conocer a los flujos de dinero que transitan por ellas. Los metodos de analisis contable y financiera ofrecen el medio de aclarar al universo de de las practicas religiosas catolicas en Francia: los ingresos proceden de varios actos rituales, y los gastos obedecen a imperativos tecnicos asi como a imaginarios religiosos. Curiosamente, parece que los catolicos que recurren a los servicios de las parroquias pertenecen a sub-grupos distintos, contiguos pero heterogeneos los unos a los otros. Si la forma del donativo es igual para todos, a causa de la reglamentacion, los feligreses regulares aparecen proporcionalmente, de acuerdo con los datos fiscales, como los mas generosos en Francia. En cuanto a los otros catolicos, resulta dificil saber si se trata de un comercio de "bienes de salvacion" o de un donativo. 